\title{
Ostension et dévolution dans l'enseignement des mathématiques
}

Anthropologie wittgensteinienne et théorie des situations didactiques

Ostension and Devolution in the teaching of mathematics

\section{Bernard Sarrazy}

\section{OpenEdition}

\section{Journals}

Édition électronique

URL : http://journals.openedition.org/educationdidactique/199

DOI : 10.4000/educationdidactique.199

ISBN : 978-2-7535-1616-8

ISSN : 2111-4838

Éditeur

Presses universitaires de Rennes

\section{Édition imprimée}

Date de publication : 1 décembre 2007

Pagination : 31-46

ISBN : 978-2-7535-0597-1

ISSN : 1956-3485

\section{Référence électronique}

Bernard Sarrazy, «Ostension et dévolution dans l'enseignement des mathématiques », Éducation et didactique [En ligne], 1-3 | Décembre 2007, mis en ligne le 01 décembre 2009, consulté le 08 décembre 2020. URL : http://journals.openedition.org/educationdidactique/199 ; DOI : https://doi.org/10.4000/ educationdidactique.199 


\title{
OSTENSION ET DÉVOLUTION DANS L'ENSEIGNEMENT DES MATHÉMATIQUES Anthropologie wittgensteinienne et théorie des situations didactiques
}

\author{
Bernard Sarrazy, \\ LACES, équipe DAESL, \\ Université Victor SegalenBordeaux 2
}

\begin{abstract}
Résumé : Ce texte présente une réflexion théorique sur le statut de l'ostension et ses rapports avec la dévolution à partir de la thèse de l'indicibilité dans la relation didactique, au croisement de l'anthropologie wittgensteinienne et de la théorie des situations didactiques. Un de ses enjeux principaux est de montrer l'intérêt de considérer la dévolution comme une nécessité didactique, nécessité liée à la faiblesse de l'ostension comme forme d'explication démonstrative («Ceci s'appelle, est un...») dans le processus didactique.
\end{abstract}

Mots Clés: ostension, dévolution, théorie des situations, règle, Wittgenstein,

\author{
À la mémoire de notre \\ ami disparu, Gilles Dumas
}

Depuis les travaux princeps de Brousseau sur le contrat, on sait que le professeur ne peut dire à l'élève ce qu'il attend de lui et ce qu'il exigera pourtant que tôt au tard il sache. Il n'a pas d'autres choix que celui de dévoluer une situation par laquelle l'élève devra voir ce qu'il ne peut que lui montrer. La tension qui naît de cet impossible à dire mais qui voudrait être dit est le point nodal de tout enseignement, et nourrit régulièrement quelques débats entre les tenants d'un certain classicisme pédagogique et ceux qui plaident en faveur de l'activité de l'élève comme une alternative possible (mais souvent mystérieuse) à cette aporie. Mais le paradoxe n'est apparent que si l'on croit que le sens de ce qui est dit et montré est contenu virtuellement dans son enveloppe signifiante et pourrait en dériver « mécaniquement », pour reprendre ici l'expression de Bouverresse (1987) à propos du paradoxe wittgensteinien de la règle. Sa réduction est alors recherchée dans des formes d'organisation pédagogique (active, classique, rationnelle, métacognitive, de l'explicitation...) qui, de ce point de vue, et malgré leurs différences apparentes, ne diffèrent pas. Telle sera la thèse qui sera défendue et présentée ici. Lidée n'est pas nouvelle : elle apparaît avec le concept de contrat de la théorie des situations, et traversera les deux périodes de la philosophie wittgensteinienne.
C'est de la rencontre de ces deux ouvres qu'a émergé, il y a une dizaine d'années, l'approche «anthropo-didactique $»^{2}$. L'originalité de ce programme est de considérer les situations d'enseignement comme le produit d'une double structuration : didactique et anthropologique. Toute situation d'enseignement se noue autour d'un projet didactique spécifique, et affecte en cela des positions aux individus dont les fonctions peuvent être étudiées indépendamment de leurs caractéristiques psychologiques, culturelles, affectives, sociales, etc. La théorie des situations est alors un instrument particulièrement puissant pour décrire ce que font effectivement ces sujets didactiques pour satisfaire le projet qui leur est dévolu (enseigner l'addition dans les entiers, résoudre un problème multiplicatif...)Mais l'action (des élèves comme celle du professeur) n'est pas entièrement réductible à sa seule dimension didactique (par exemple, les formes d'organisation de l'enseignement ne sont que très rarement justifiées par des motifs didactiques: « je pratique le travail de groupe car j'estime que les élèves doivent apprendre à travailler ensemble. »; les positionnements des élèves à l'égard des implicites du contrat s'avèrent fort variables selon les contextes familiaux ou scolaires...). Cette seconde dimension, qualifiée d'anthropologique, 
ouvre sur tout un ensemble de dimensions (comme les croyances, les valeurs, les idéologies...) qui déterminent (non causalement) la manière de faire ce qui est fait (songeons par exemple aux divers jeux de langage spécifiques du monde scolaire : répondre à une question, solliciter la parole ou la prendre, organiser un débat...). Bref, si les situations scolaires imposent aux élèves comme aux professeurs des rôles indépendants de leurs caractéristiques sociales et culturelles (dimension didactique), la manière de les interpréter (au sens théâtral du terme) est à rechercher dans la façon dont elles sont « vues» par les sujets, vision, qu'en référence à Wittgenstein, j'appellerai Arrière-plan. L'Arrière-plan, dit Wittgenstein «ce n'est pas ce qu'un individu est en train de faire, c'est cet ensemble grouillant; c'est lui qui détermine notre jugement, nos concepts et nos réactions » (Wittgenstein, 1994, § 629). Nous l'avons dit, le cadre d'analyse de cette approche est redevable à la fois à la théorie des situations didactiques de Brousseau (1998) et à l'anthropologie wittgensteinienne. Ce texte sera aussi l'occasion d'en présenter quelques aspects en rapport avec la question de l'indicibilité ${ }^{3}$.

\section{Brousseau, Wittgenstein : une thèse commune}

« Le savoir et le projet d'enseigner vont devoir s'avancer sous un masque. [...] si le maître dit ce qu'il veut, il ne peut plus l'obtenir. » G. Brousseau « Tout ce qui peut être dit peut être dit clairement; et ce dont on ne peut parler, il faut le passer sous silence » L. Wittgenstein

$\mathrm{Au}$ début des années soixante, Guy Brousseau, alors jeune instituteur, rentre du service militaire; dans ses poches, quelques carnets sur lesquels étaient consignés quelques scénarios de leçons «non-verbales », comme il les appelait. Si l'intuition selon laquelle les connaissances ne pouvaient pas se transmettre 'directement' était présente, les diverses réponses disponibles dans la noosphère (de Freinet à Polya en passant par Diénès et d'autres) ne le satisfaisaient pas. Comment transmettre une pratique mathématique, une manière d'agir, de prouver, de considérer les problèmes et de rechercher à les résoudre?... La théorie des jeux et les travaux du logicien Lorenzen lui inspireront l'idée initiale du diptyque proposant-opposant - qui deviendra ulté- rieurement le schéma de base de la dialectique de la validation. Mais si en théorie des jeux le proposant et l'opposant sont censés disposer des connaissances requises pour débattre, ce n'est pas le cas pour les élèves; étudier des conditions susceptibles de caractériser ces connaissances et de les modéliser sous forme de situations s'imposa alors comme une nécessité $^{4}$. C'est à l'occasion du congrès de l'APMEP de Clermont-Ferrand en 1970 que Brousseau en présentera la première formulation (cf. Brousseau, 1972) ; la rupture avec une conception indifférenciée de la notion d'activité (fort en vogue à l'époque) était consommée. Si les piagétiens pouvaient reconnaître dans les trois dialectiques l'influence genevoise, ils se trompaient car l'essentiel n'était pas là : même si la théorie des situations faisait écho à la conception adaptative de la connaissance de Piaget ${ }^{5}$, la théorie de l'équilibration ne permettait ni d'étudier, ni ( $a$ fortiori) de définir les propriétés des milieux susceptibles de poser des problèmes effectifs aux élèves et dont le dépassement exigeait des adaptations correspondant aux connaissances à enseigner. Ces propriétés ne peuvent ni être déduites seulement de l'élève (pris comme un sujet épistémique ou psychologique), ni seulement des dispositifs dans leur épaisseur pédagogique, ni encore dans le seul examen de la connaissance elle-même, mais bien dans les jeux complexes des rétroactions apparaissant dans le milieu comme une sorte de nervure mathématique, pour reprendre ici l'expression de G. Dumas. Ces rétroactions doivent être tout à la fois suffisamment lisibles ('assimilables') par l'élève mais aussi suffisamment informatives afin d'autoriser les régulations constructives par lesquelles il sera possible de reconnaître que les élèves, par les transformations de leurs décisions à l'égard du milieu, apprennent des mathématiques. C'est donc fondamentalement d'un point de vue anthropologique que Brousseau posa la question de la diffusion des connaissances mathématiques : étudier les propriétés des situations susceptibles de réunir les conditions pour re-créer une activité mathématique. Tel est un des apports majeurs de cette théorie à l'enseignement des mathématiques : rapporter la question du sens des connaissances à leurs usages via les situations vs produire des situations permettant l'usage des connaissances pour en 'montrer' le sens. La nuance peut paraître byzantine mais elle est fondamentale, et c'est probablement dans ce renversement que la convergence avec l'anthropologie wittgensteinienne est la plus manifeste. 
Quelques décennies plus tôt, en 1919, Wittgenstein, alors âgé de 30 ans, rentre de Monte Cassino, où il était prisonnier. Dans ses poches, quelques notes (Les Carnets, 1971) qui deviendront le retentissant Tractatus Logico Philosophicus (1961) se refermant sur le célèbre aphorisme « Sur ce dont on ne peut parler, il faut garder silence » (trad. de Granger, 1993). Ce manuscrit ${ }^{6}$, rédigé en large part sur le front d'Italie, va susciter un vif débat au sein de l'Ecole néo-positiviste du Cercle de Vienne. Certains membres, comme son fondateur Schilck et son assistant Waismann, l'érigeront comme bible de l'empirisme logique. Le Tractatus constituera, quelques années plus tard, sa thèse de doctorat (le 18 juin 1929) et sera publié avec une introduction de Russell, désavouée par Wittgenstein ${ }^{7}$. C'est au moment où son manuscrit est en passe d'être publié, au moment même où il est régulièrement sollicité par le Cercle de Vienne, et après s'être déshérité d'une fortune colossale au profit de ses frères et sœurs et des artistes, qu'il décide de rentrer, en 1922, à l'Ecole de formation des Instituteurs de la Kundmanngasse à Vienne ${ }^{8}$. Durant les six années que dure sa carrière d'instituteur dans divers petits villages de Basse-Autriche, il participe activement au mouvement de réforme de l'enseignement impulsé par le ministre de l'éducation Otto Glöckel, dont il partageait certains principes propres aux pédagogies nouvelles - comme celui d'encourager l'élève à réfléchir par lui-même. L'importance qu'il accordait à l'enseignement des mathématiques lui faisait consacrer les deux premières heures de la matinée à cette matière (Monk, 1993, 197-8) ; «il ne cherchait pas à améliorer 'de l'extérieur', mais 'à l'intérieur'. Il voulait développer l'intelligence des enfants en leur enseignant les mathématiques. » (id., 193). Son expérience pédagogique prit fin suite à une cabale organisée par les habitants d'Otterthal et par certains de ses collègues, plus motivée par des raisons politiques, qu'attachées à sa forte personnalité pédagogique. Cette conspiration clôtura définitivement sa carrière d'instituteur. "Il jeta donc la bouillotte » en avril 1926, comme il l'avait confié à Keynes :

« [Je resterai instituteur] tant que je sentirai que les problèmes que je me crée de la sorte peuvent m'être bénéfiques [...] Si l'on a mal aux dents, il est bon d'appliquer une bouillotte chaude sur son visage, mais cela n'a d'effet que dans la mesure où la chaleur vous fait un peu mal. Je jetterai la bouillotte lorsqu'elle ne me donnera plus la douleur particulière qui fait du bien à mon caractère. » (Wittgenstein, courrier à Keynes cité par Monk, 1993, 231).

C'est une conférence de Brouwer sur la critique du logicisme de Russell, prononcée à Vienne en mars 1928, intitulée « La mathématique, la science et le langage », qui ramènera Wittgenstein à la philosophie. Dès le début de ce qu'il est d'usage d'appeler sa seconde période, Wittgenstein reviendra sur des points essentiels du Tractatus : il abandonnera la méthode de l'atomisme logique au profit de l'étude du langage ordinaire - intérêt probablement lié à son expérience pédagogique (songeons par exemple à l'importance accordée dans les Investigations aux thèmes de l'enfant et de l'apprentissage - cf. Clanché, 1994) ${ }^{9}$. Mais une thèse centrale perdurera : l'indicibilité des relations sémantiques. Cette thèse, considérée comme l'une des plus fortes du Tractatus, fondée sur la célèbre distinction " dire / montrer », désavouera, comme le pointe Chauviré $(1989,90)$, la théorie des types logiques de Russell, mais aussi, par avance, l'entreprise de Carnap dans sa Syntaxe logique du langage.

Wittgenstein, Brousseau. Le premier est philosophe; le second, didacticien; tous deux, passionnés par les mathématiques et leur diffusion, les ont enseignées à des jeunes enfants. Leur intérêt pour une approche non-mentaliste des questions de sens, de compréhension et d'apprentissage constitue un point de rencontre original des deux ouvres que nous rapprocherons ici sur une idée force : l'indicibilité des relations sémantiques dans ses rapports avec la genèse de la signification ${ }^{10}$.

\section{La méthode anthropologique}

Les mathématiques sont un phénomène anthropologique. Wittgenstein $(1983,318)$.

La thèse de l'ineffabilité des relations sémantiques, commune aux deux auteurs, peut être vue comme un ancrage anthropologique consistant à dé-mentaliser des questions traditionnellement étudiées par la psychologie (comme celles de signification, d'apprentissage et de compréhension) en les rapportant à notre univers humain, historique et culturel. Examiner les conditions susceptibles de montrer ce qui ne peut être dit, décrire les conditions de la genèse d'une 
pratique par et dans laquelle les mots, les concepts... et les savoirs peuvent prendre sens, constitue certainement la méthode commune à Wittgenstein et à Brousseau. Refuser l'un comme l'autre toute autre forme d'explication que le consensus dans l'action comme critère de la compréhension, permet de se passer des concepts mentalistes tels ceux de dispositions ${ }^{11}$ ou de représentations mentales (cf. Sarrazy, 2002) : la compréhension (d'un mot, d'une règle, d'un algorithme... bref d'un langage) a toujours partie liée avec l'usage, à la fois comme instrument et critère de l'apprentissage, dans une forme de vie (chez Wittgenstein) ${ }^{12}$, dans un milieu (chez Brousseau) sans lequel la pratique ne serait que simple activité, pour reprendre ici la distinction établie par Conne (1999).

Mais la convergence des deux ouvres n'est pas seulement épistémologique, elle est aussi dans la méthode: pour Brousseau, toute connaissance est modélisable par une situation qui permet d'en préserver le sens; pour Wittgenstein «le sens d'une question, c'est la méthode pour y répondre [...] Dis-moi comment tu cherches et je te dirai ce que tu cherches. » (Wittgenstein, 1975, 66). Cette méthode, qualifiée par Rhees (1992) d'anthropologique, consiste à imager des situations pour clarifier des questions de signification; elle s'est avérée particulièrement productive tant en philosophie qu'en didactique.

Pour Wittgenstein, c'est l'examen des formes de vie : ce sans quoi il serait même impossible d'imaginer un langage ( Si un lion pouvait parler, nous ne pourrions le comprendre. Même si quelqu'un exprimait tout 'ce qui est au-dedans de lui', nous ne pourrions le comprendre. », 1961, 356). Débusquer les pièges que nous tend le langage en décrivant les conditions dans lesquelles les propositions peuvent signifier, tel est le projet wittgensteinien : « signifier l'indicible, en représentant clairement le dicible » (1961, § 4.115).

Modéliser les pratiques des mathématiciens pour envisager les conditions de leur re-production, telle fut l'idée princeps de la théorie des situations. Ainsi, ces phénomènes que l'on nomme "apprendre ", " comprendre ", " mesurer», " calculer mentalement $» . .$. n'ont de sens que rapportés à la manière dont les objets sur lesquels ils portent sont pris dans un type de pratiques spécifiques dans des circons- tances déterminées mais non déterminables a priori. $\mathrm{Nul}$ besoin de rechercher d'autres fondements à ces pratiques que la manière dont elles ont été enseignées ( « Enseignez-nous la, disait Wittgenstein à propos des mathématiques, et vous l'aurez fondée »). C'est dans cette irréductibilité radicale de l'usage et du sens, du sémantique et du syntaxique, que réside la thèse la plus explosive chez Brousseau et Wittgenstein. D'ailleurs, la confusion possible sur l'auteur des quelques lignes pourrait en témoigner :

Lélève reçoit une règle et des exemples, et le maître peut pour sa part dire qu'il veut dire quelque chose qui, bien que non énoncé, est transmis indirectement au moyen des exemples. Mais le maître lui aussi n'a que la règle et les exemples. [...] C'est une illusion de croire que l'on produirait la signification dans l'esprit de quelqu'un par des moyens indirects, à travers la règle et les exemples. (Wittgenstein, 1992, 161.).

Le caractère indicible de ce qui doit être appris me paraît assez souvent sous-estimé (alors que j'aurais tendance à le placer à l'origine des didactiques ${ }^{13}$ ) ; au mieux, l'indicibilité est considérée comme une sorte de coquetterie épistémologique intéressante, mais pas assez pour susciter quelques vigilances épistémologiques sur l'usage de certains concepts (songeons par exemple à l'usage de quelques expressions telles " connaissances disponibles», " manipuler le contrat $» . ..)^{14}$; au pire on fait comme si tout pouvait être dit, montré, ou dériver mystérieusement de l'activité de l'élève (Sarrazy, 2002a). Le courant méta-cognitif qui s'est imposé à partir des années quatre-vingt, le développement important du cognitivisme, constituent certainement des exemples les plus manifestes de cette négation. Mais ce n'est pas cet aspect que nous discuterons ici (nous l'avons déjà fait par ailleurs, Sarrazy, 1995, 1997). Nous développerons la thèse de l'indicibilité à partir de la tension qui naît entre le désir ostensif du professeur, désir contingentement incitatif ${ }^{15}$ de dire et de montrer ce qu'il attend des élèves, et l'impossible dérivation du sens (d'un usage) de cette seule forme d'explication démonstrative. Il ne s'agira pas de réhabiliter l'ostension comme procédé didactique (comme le réclament certains aujourd'hui), mais de montrer qu'elle correspond à une sorte de " mal nécessaire » constitutif de l'enseignement, et que l'essentiel est moins dans l'ostension elle-même que dans les fonctions qu'elle est censée assurer dans la relation didactique ou les effets que l'on peut en espérer ${ }^{16}$. 


\section{Le nomadisme de l'ostension}

Lostension est souvent présentée comme le symptôme d'une idéologie pédagogique dont l'empirisme constituerait la source. C'est le cas de Fregona pour qui « l'idéologie empiriste procure des solutions faciles à mettre en ouvre dans les décisions didactiques » $(1995,155)$. C'est aussi à ce titre que l'ostension fut vivement condamnée par Aebli (1966, 7) dans sa critique du sensualisme-empirisme qui inspira la didactique du même nom. Considérée comme porteuse de présupposés inductivistes, euxmêmes renforcés par les contraintes effectives de la relation didactique, Ratsimba-Rajohn la place lui aussi sous le joug d'une conception de la connaissance « comme seulement un système d'affirmations : règles, procédures, algorithmes, méthodes, procédés, définitions, etc. Cette conception impliquerait une activité enseignante qui consiste essentiellement à pratiquer un transfert plus ou moins subtil et judicieux de la connaissance principalement conçue comme un système d'affirmations à mémoriser et à reproduire. » $(1992,86)$.

Mais s'agissant des mêmes observations, avec quasiment la même structure argumentative, ne pourrait-on pas également affirmer que l'ostension a partie liée avec l'idéalisme? On ne peut manquer de constater la relative proximité entre la définition qu'en donne Ratsimba-Rajohn et ce que disait Platon des êtres mathématiques indépendants du monde sensible:

Le rapport du sujet et de l'objet nouveau est institué seulement au niveau de la représentation de l'objet présumé indépendant du sujet [...] on suppose que la conception de la notion n'est qu'un résultat nécessaire (donc automatique) de sa perception : toucher, voir, modifier... [...] les procédés ne permettent que des interactions limitées, superficielles, ou familières avec les moyens tels que : image, schéma, graphe, discours, liste de propriétés ou d'axiomes. Les enseignants prétendent ainsi fournir 'd'un coup' tous les éléments et les relations constitutifs de la notion visée. Ce sont de tels procédés que nous qualifions d'introduction ostensive et que nous appelons ostension. » (Ratsimba-Rajohn, 1977, 7-8).

[Les géomètres] font en outre usage de figures visibles et, sur ces figures, ils construisent des raisonnements, sans avoir dans l'esprit ces figures elles-mêmes, mais les figures parfaites dont celles-ci sont des images, raisonnant en vue du carré en lui-même, de sa diagonale en elle-même, mais non en vue de la diagonale qu'ils tracent; et de même pour les autres figures. (e) - Celles qu'ils façonnent et peignent, objets qui produisent des ombres ou qui se réfléchissent à la surface de l'eau, à leur tour elles sont traitées par eux comme des copies quand ils cherchent à voir les figures absolues, objets dont la vision ne doit être possible pour personne (a) autrement que par le moyen de la pensée. [...] chez celui qui contemple, la contemplation par l'esprit est bien l'œuvre d'une discursion forcément relative à des objets pris en eux-mêmes et sans recours aux sensations. (Platon, République VI, 510e, 511a, 511c)

Pour des motifs différents de l'empiriste, l'idéaliste flirte aussi avec l'ostension. Le platonisme mathématique ${ }^{17}$ n'affirme-t-il pas l'existence d'objets mathématiques indépendante de leurs usages et du monde sensible? N'emploie-t-on pas indifféremment montrer et démontrer? Songeons aussi à l'impatience de certains professeurs lorsque leurs élèves ne voient pas que tel problème relève de tel ou tel type de démonstration? Laissons cela car la question de l'existence des objets mathématiques est ici moins fondamentale que celle de leur forme d'existence. L'existence de $\pi$ est toute aussi réelle que celle d'un biberon et il n'y aurait aucun sens à la mettre en doute. Ces objets, qu'ils soient sensibles ou idéels, sont tout aussi réels les uns que les autres par les effets qu'ils engendrent, et ne diffèrent entre eux que par leurs formes d'existence dans une forme de vie, par les pratiques spécifiques qu'ils suscitent ${ }^{18}$ et par le travail des institutions qui en garantissent l'efficacité potentielle - le " potentiel d'action » dont parle Rouchier $(1996,194)$. Ce sont d'ailleurs ces formes d'existence, plus que les objets eux-mêmes, qui sont au centre des questions didactiques (cf. Sarrazy, 2005), et l'histoire des savoirs est didactiquement moins productive que l'ingénierie ou la théorie des situations pour examiner les conditions de la transposition, non de ces objets, mais bien des pratiques, via des dispositifs didactiques entre diverses institutions. Et c'est bien en cela que réside l'essentiel du travail du professeur tel que le décrit Brousseau :

Le professeur doit simuler dans sa classe une micro société scientifique s'il veut que les connaissances soient des moyens économiques pour poser de bonnes questions et pour trancher des débats, s'il veut que les 
langages soient des moyens de maîtriser des situations de formulation et que les démonstrations soient des preuves. Mais il doit aussi donner les moyens à ses élèves de retrouver dans cette histoire particulière qu'il leur a fait vivre, ce qu'est le savoir culturel et communicable qu'on a voulu leur enseigner. (Brousseau, 1998, 49-50).

Mais si l'idéalité de ces objets est une illusion, elle n'est pas illusoire; elle est nécessaire au fonctionnement des institutions - nous en faisons l'expérience chaque fois que nous nous arrêtons au feu rouge. C'est par leur usage, parce qu'ils autorisent ou interdisent, que réside leur sens : comme pour la règle, ce n'est ni leur définition, ni la mémorisation de ce qu'ils rendent possible qui permettra de saisir leur signification. En effet, 'connaître la règle' et 'savoir jouer' sont deux choses différentes : la règle renvoie toujours à un au-delà et recèle toujours un degré de liberté irréductible ${ }^{19}$. C'est cette part d'indétermination qui détermine tout à la fois la liberté du sujet et sa responsabilité à l'égard de son usage, car la règle ne peut le renseigner à tout instant puisque, comme le poteau indicateur auquel la compare Wittgenstein ${ }^{20}$, elle ne contient pas en ellemême ses propres conditions d'application.

Toute explication comporte donc une dimension ostensive, et entretient en cela cette part d'illusion :

[Lorsqu'on explique un concept] on donne des exemples et on veut qu'on les comprenne dans un certain sens. - Or par cette expression je n'entends pas qu'il doive voir dans ces exemples l'élément commun que - pour une raison quelconque - je ne pouvais exprimer. Mais j'entends qu'il utilise désormais ces exemples d'une manière déterminée. Le fait de donner des exemples n'est pas ici un moyen indirect de l'explication, - faute de mieux. Car toute explication générale peut donner lieu à des malentendus. Voilà comment nous jouons le jeu. (Wittgenstein, 1961, § 71).

Sans cette illusion collective de l'idéalité des objets, de la généralité de la règle, de la communicabilité de ce qui est commun aux cas particuliers, le langage cesserait, nous dit Wittgenstein («S'il n'y avait aucune unanimité au sujet de ce que nous appelons 'rouge' le langage cesserait », 1983, III, $\S 70$ ). Didactiquement, la nuance est importante car elle nous invite à distinguer deux conceptions de la transposition et corrélativement, deux conceptions du contrat : "'Transposition d'objets' / 'transposition de pratiques' ", « 'définition d'un contrat' / 'recherche d'un contrat' ${ }^{21}$. On oublie trop souvent, remarque Conne $(1999,59)$, que le fait que l'enseignant soit détenteur et dépositaire du savoir à transmettre «n'est qu'une condition à l'entreprise et qu'au delà, tout reste à faire. », et c'est fondamentalement ce 'faire', ou plutôt ce 'faire faire' en vue de 'faire voir' qui doit nous préoccuper. On comprend mieux en quoi il nous semble davantage fécond, pour une meilleure compréhension des effets de l'ostension, de s'interroger sur les conditions de possibilité de ces pratiques qui engagent des mathématiques, que sur les éventuelles conceptions épistémologiques susceptibles de les caractériser, voire de les inspirer.

Empirisme, idéalisme, réalisme, constructivisme... ne seraient que les formes savantes pour décrire les pratiques d'enseignement, et correspondent rarement aux blasons pédagogiques que les professeurs arborent lorsqu'on les interroge sur le sens qu'ils accordent à leur propre décision. Donnons l'exemple des déclarations de ces professeurs dont les milieux didactiques, pourtant, ne diffèrent que par quelques traits de surface:

Moi, je ne fais jamais des leçons classiques! Je mets habituellement les élèves en groupe ils ont une situation problème à résoudre, donc ils inventent une solution pour la résoudre. On compare, j'envoie quatre ou cinq gosses au tableau : pof pof pof! On compare les solutions, comment on a fait ça. On critique, c'està-dire on analyse et puis après on se met d'accord sur les meilleures...

J'essaie de trouver des situations de recherches, de découvertes où les élèves essaient de... et des situations d'affrontements entre gamins, pour leur permettre de construire des savoirs entre eux, de leur proposer des hypothèses pour qu'ils voient entre eux, si elles sont justes ou fausses; ensuite moi je sers, à la fin, en dernier ressort, de juge pour voir un petit peu celui qui a raison ou tort.

Moi, je ne fais pas de leçon traditionnelle. Je les mets devant un exercice, une situation, et on analyse cette situation et ensuite je propose d'autres exercices semblables qu'ils doivent résoudre d'après ce que nous avons fait... il est rare que je fasse une leçon type leçon traditionnelle. 
Pour les problèmes, je veux une phrase de réponse. Ce sur quoi j'insiste beaucoup c'est sur les mécanismes parce que, avec les mécanismes, c'est cent pour cent de réussite, même pour les plus faibles. C'est quand même agréable qu'aux gosses, on puisse de temps en temps leur dire : «Mon vieux, ce soir, c'est parfait, vraiment parfait quoi!»

Certes ces blasons pédagogiques ont des effets didactiques manifestes (cf. Roiné, 2007; Marchive, 2007; Sarrazy, 2001, 2002b), mais ne sont pas aussi didactiquement déterminants qu'on pourrait le croire spontanément. Le professeur n'est pas entièrement maître dans sa propre classe, et l'exigence didactique prend le plus souvent le pas sur les convictions, les opinions ou les croyances.

[Imaginons deux individus] L'un est un réaliste convaincu, l'autre un idéaliste convaincu, et chacun éduque ses enfants en conséquence. Ils ne veulent rien leur enseigner de faux sur une question aussi importante que celle de l'existence ou de la non existence du monde extérieur. [...] Mais l'idéaliste enseignera quand même à ses enfants le mot 'fauteuil', car il veut sans aucun doute leur apprendre à faire diverses choses, par exemple aller chercher un fauteuil. Où donc sera la différence entre ce que diront les enfants élevés à la manière idéaliste et les enfants élevés à la manière réaliste? Ne se résumera-t-elle pas dans la différence entre les cris de guerre? (Wittgenstein, 1994, § 338-339).

Ainsi ne pourrions-nous pas réinterpréter l'opposition introduite par Salin et Berthelot entre " ostension assumée » et "ostension déguisée » 22 en considérant ces deux modalités de l'ostension comme une simple différence entre les «cris de guerre » pédagogiques des professeurs? C'est l'idée que nous soutiendrons dans la suite de ce texte.

\section{Le 'voir comme' : une alternative aux ornières du mentalisme}

Expliquons le mot 'tove' en montrant du doigt un crayon et en disant 'ceci est tove' [...] La définition ostensive "ceci est tove' peut-être interprétée de bien des manières [...] ; 'Ceci est un crayon', 'Ceci est rond', 'Ceci est du bois'... Wittgenstein, 1965, 36.
Dans la conception du contrat classique, le professeur montre la règle (selon diverses mises en scène plus ou moins "assumées », plus ou moins "déguisées »), à charge de l'élève d'en repérer l'usage: de ce 'montré' doit surgir un 'vu'. Mais qu'est-ce qui est effectivement montré et qu'est-ce qui doit effectivement être vu?

Jean à Eva (3 ans): Maintenant je vais te mettre tes chaussures.

Eva : Non, ce ne sont pas mes chaussures, ce sont mes chaussons!

Jean : Ah oui, tu as raison : je vais te mettre tes chaussons. Donne-moi ton pied.

Eva : On dit « pardon»!

Jean : On dit « pardon»? Pourquoi?

Eva : Quand on se trompe, on dit «pardon»!

Trois jours avant, son père s'était repris en s'adressant à elle et, comme souvent dans ce cas-là, avait ponctué machinalement sa reprise par un "pardon». Eva, surprise, lui avait demandé les raisons de ce "pardon"; naturellement, il lui en justifia l'usage par le fait qu'il avait voulu dire A et non B. "Vouloir dire " et "se tromper" ne sont pas encore pour Eva des jeux de langage distincts pour dissocier ces deux usages $d u$ " pardon ».

Lostension est à la dévolution ce qu'est l'explicitation au contrat : elle désigne toujours un au-delà qui veut être montré mais qui ne peut être vu que par celui qui sait (c'est souvent à ce « vu » que l'on voit que l'élève a appris). C'est l'acceptation de ce renoncement à «faire voir » qui justifie la recherche de conditions didactiques favorables à l'enseignement et qui, étonnamment, fonde la dimension pédagogique de la relation à l'élève ${ }^{23}$ puisqu'elle l'instaure dans un topos déterminé et non déterminant (au sens causaliste du terme) :

Est-ce que je me soucie de l'intériorité de celui à qui je fais confiance? Si je ne lui fais pas confiance, je dis 'je ne sais pas ce qui se passe en lui'; mais si je lui fais confiance, je ne dis pas que je ne sais pas ce qui se passe en lui. Si je ne me méfie pas de lui, je ne me soucie pas de ce qui se passe en lui. (Les mots et leur signification.) Dans une conversation normale, je ne me soucie pas de la signification des mots, de ce qu'il y a derrière eux. Les mots coulent et le passage se fait de lui-même entre eux et les actes, entre les actes et eux. (Wittgenstein, 1994,§ 602-603). 
On voit bien comment les questions habituellement associées à l'usage mentaliste de l'ostension (un voir + un pensé) relayées, le plus souvent implicitement, par des propositions didactiques imprégnées de psychologie ${ }^{24}$, engagent les professeurs sur de fausses pistes en orientant leur regard sur des dimensions qu'ils ne peuvent ni atteindre (l'intériorité), ni traiter (la pensée). Rien ne s'oppose alors à l'interprétation des difficultés des élèves comme le fait d'un destin inéluctable; comme l'écrit Solère-Queval : « comment se révolter contre les inégalités si elles sont perçues comme naturelles? On fera donc croire aux hommes que certains ont une âme faite d'or, d'autres d'argent, d'autres enfin de fer et d'airain. La distribution des fonctions sociales sera dès lors fondée en nature, nous dirions aujourd'hui biologiquement. Il ne serait pas difficile, en effet, de transposer ce récit dans le vocabulaire de la génétique ou de la psychologie modernes, les chromosomes et gènes remplaceraient les métaux et le QI dirait la teneur en métal d'un tel alliage! » (Solère-Queval, 2002, 4).

Le «voir comme» wittgensteinien permet d'échapper à cet écueil. Il ne s'agit plus d'un 'voir' auquel viendrait se surajouter un élément non sensoriel (une pensée, un certain état mental, une représentation...) mais duquel émergerait la 'naissance d'un aspect' - elle correspond à l'expérience que nous faisons lorsque nous apercevons par exemple une ressemblance entre deux visages (Wittgenstein, 1985, § 431). Lorsqu'on nous y invite, cette impression visuelle peut être 'forcée' par l'imagination ou la volonté (voir un animal dans un nuage), par le savoir (voir un triangle en considérant un segment particulier comme étant sa base, puis en considérant un autre segment)... mais elle ne peut être montrée (au sens de l'ostension classique (et donc exigée) puisque le perçu est resté invariable (id., § 440). Les modalités de l'ostension (qu'elle soit assumée ou déguisée) sont ici moins fondamentales pour saisir le brouillage didactique attaché à la confusion liée à l'amalgame de ces deux usages du « voir » : décrire la vision de l'aspect dans les mêmes termes que la vision d'un état ${ }^{25}$. La plupart des effets de contrat, et plus particulièrement ceux correspondant aux phénomènes de sensibilité (voir ceci comme cela), se dissipent grâce à cette distinction. On pourrait avantageusement la rapprocher des rapports entre règle et action chez Wittgenstein : " Gardons-nous de concevoir la signification comme un rapport occulte entre les mots et choses, et de penser que tous les usages d'un mot sont contenus dans ce rapport, de la même façon que l'on peut dire que la graine contient l'arbre. »
(Wittgenstein, 1965), « Les mots sont comme les glands : un chêne peut en sortir. » (Wittgenstein, 1990, 69). Et nul besoin de considérer que l'élève voit ce qu'il convient de faire dans cette situation; il agit conformément (ou non) à la règle et non en vertu de ce qu'elle exige. Il 'sait' comment il doit agir pour faire ce qu'il fait sans pour autant avoir besoin de postuler qu'il est là en train 'd'appliquer des règles'.

\footnotetext{
«Imagine un processus dans lequel un homme qui pousse une brouette s'est aperçu qu'il lui faut nettoyer l'axe de la roue lorsque la brouette est trop dure à pousser. Je ne veux pas dire qu'il se dit à lui-même: Chaque fois que la brouette est trop dure à pousser... Il agit simplement de cette façon. » (Wittgenstein, 1983, § 317) même si, ajoute$\mathrm{t}$-il, ce même homme pourra dire à son ami qui pousse une brouette qui a du mal à avancer 'Eh oh! Nettoie l'axe de ta roue!'.
}

Prenons un exemple; examinons la situation suivante:

Un professeur montre le schéma suivant à son élève :

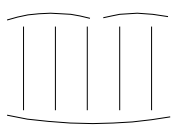

Nous avons ici quelque chose qui nous paraît inexorable. Et pourtant : l'inexorabilité ne peut se trouver que dans les conséquences! Sinon ce n'est qu'une image. (Wittgenstein, 1983, I, § 62).

C'est bien la naissance d'un aspect qui est attendu par le professeur (une manière particulière de 'voir' 5) et non la vision d'un état ( 5 barres et 3 traits). Le caractère inexorable de ce qui est montré (« Tu dois voir 5 comme 3 et $2 »$ ) ne peut dériver de l'expérience perceptive à laquelle se surajouterait une signification, puisque la nécessité qui s'exprime n'est pas contenue dans le perçu, mais bien dans la manière dont le professeur procède pour le montrer (manière de considérer le dessin, de se comporter à son égard...). Indépendamment de son blason, le professeur naturalise cette inexorabilité en la considérant comme acquise, par une sorte d'appel à la complicité de l'élève que j'aurais tendance à rapprocher de ce dit Chevallard à propos de «l'appel à 
la complicité dans la reconnaissance ontologique; l'existence de l'objet apparaît alors comme évidente, non douteuse, plus justement non susceptible de doute; l'objet est installé, par la monstration qui le désigne dans son existence entêtée, dans un état qui échappe au questionnement, parce que tout questionnement le suppose. » $(1991,91)$. Je situerai cet appel à la complicité au lieu même de l'articulation des dimensions non-didactiques et didactiques de la dévolution, lieu où se mêlent croire et savoir, soudés par la confiance dont nous parlions ci-dessus, cette phase que Conne et Brun désignent comme 'phase d'installation' : « le début d'un enseignement n'est pas [...] une phase de construction, mais une phase d'installation, de mise en présence de l'élève face à l'objet à apprendre. La conceptualisation ne peut que succéder à l'initiation. [...] Le contrôle du dispositif d'enseignement s'établit bel et bien à la faveur d'un double déjà là, sémantique puis fonctionnel. On le voit, ce contrôle par le dispositif d'enseignement se paie au prix d'une interposition dans le rapport de l'élève à la réalité, la connaissance de ce dernier est sous tutelle d'une réalité instituée. " (Conne, Brun, 1990). Dans cette phase, il n'y a pas de place au doute de l'élève; imaginons avec Wittgenstein : « Maître et élève. Lélève ne s'ouvre à aucune explication car il interrompt continuellement le maître en exprimant des doutes, par exemple quant à l'existence des choses, la signification des mots, etc. Le maître dit : 'Ne m'interromps plus et fais ce que je te dis; tes doutes, pour le moment, n'ont pas de sens du tout. [...] L'enfant apprend en croyant l'adulte. Le doute vient après la croyance.» (Wittgenstein, 1976, $\S 160 \& \S 310)^{26}$.

La conception dualiste de l'ostension (un 'voir' et un 'penser') ne permet pas de décrire ce qui se passe dans cette situation et reste impuissante pour ouvrir les yeux de l'élève à la signification :

Celui qui est incapable de comprendre ou d'employer la locution 'voir ce signe comme une flèche' celui-là je le nomme 'aveugle à la signification'. Il n'y aurait aucun sens à lui dire 'Il faut que tu essayes de le voir comme une flèche', et ce n'est pas de cette manière qu'on pourra l'aider. (Wittgenstein, 1989, § 334).

La convergence avec le conventionnalisme non contractuel ${ }^{27}$ de la théorie des situations, et plus particulièrement avec le paradoxe de la dévolution, est ici particulièrement manifeste :
Le maître veut que l'élève veuille ne tenir la réponse que de lui-même mais en même temps il veut, il a le devoir social de vouloir, que l'élève donne la bonne réponse. Il doit donc communiquer ce savoir sans avoir à le dévoiler, ce qui est incompatible avec une relation contractuelle. (Brousseau, 1998).

"Le maître veut que l'élève veuille...», d'aucuns souriraient de la formule, mais le paradoxe est tenace et ne se laisse pas réduire par les naïves explicitations des enjeux du contrat que par ailleurs ils proposent. Certes, le professeur peut exiger que l'élève reproduise ce qu'il lui a « dit » ou « montré », il peut aussi lui demander de mémoriser ou de répéter les règles, les définitions ou les solutions types, mais il ne peut le sommer d'apprendre en exigeant de lui qu'il reconnaisse ici et maintenant une des occasions d'emploi de la règle enseignée, même s'il a le sentiment qu'il pourrait lui en révéler l'aspect par quelques « cas particuliers » judicieusement choisis :

'Qu'est-ce que la longueur?', 'Qu'est-ce que le sens?', 'Qu'est-ce que le nombre un?', etc., toutes ces questions provoquent en nous une crampe mentale. Nous sentons que nous ne pouvons rien montrer en réponse, et que pourtant nous devrions montrer quelque chose. (Wittgenstein, 1965, 35).

Telle est la tension qui structure le contrat et qui fonde épistémologiquement la dévolution. Trop souvent considérée comme un impératif constructiviste, forme moderne ou euphémisée des pédagogies actives, elle est le lieu où se manifeste la volonté du maître de montrer quelque chose à l'élève ${ }^{27}$, et la nécessité de réunir les conditions susceptibles de faire naître une manière de voir à l'élève ${ }^{28}$.

\section{Conclusion}

Si nos élèves apprennent les mêmes mathématiques, il n'est pas sûr qu'ils apprennent tous la même manière d'en faire. Ce qui est montré, enseigné et retenu est moins essentiel que la manière dont on leur a fait considérer les problèmes, dont on les a conduits à chercher à les résoudre... car ce qui doit être appris ne peut pas s'enseigner (quelles que soient les formes que peut prendre cet enseignement). Ce sont en effet par ces manières de faire faire des mathématiques que l'enfant apprend à voir ce qu'on 
ne peut lui montrer (« L'enfant n'apprend pas qu'il y a des livres, qu'il y a des sièges, etc., mais il apprend à aller chercher des livres, à s'asseoir sur un siège, etc. » Wittgenstein, 1976, § 476) : telle était, rappelons-le, l'intuition fondamentale de Brousseau. C'est l'universalité de ces manières de voir, d'agir, de chercher... que décrète l'institutionnalisation, l'expression d'un usage commun auquel désormais doivent se contraindre les élèves. Elle manifeste la volonté de la création d'une unanimité : "Maintenant, nous avons la même façon de voir et de parler! Et vous pouvez (devez) maintenant poursuivre tout seuls. ».

«L'effet du 'et ainsi de suite' sera de créer l'unanimité au-delà de l'enseignement. Il est donc déterminé que tous ou presque nous comptons et calculons de façon identique. [...] Pourrait-il exister une arithmétique sans unanimité entre ceux qui comptent? Un homme seul pourrait-il compter? Un homme seul pourrait-il suivre une règle? Ces questions sont-elles similaires à celleci : 'Un homme seul peut-il faire du commerce?' II n'y a de sens à dire 'et ainsi de suite' que lorsque 'et ainsi de suite' est compris. C'est-à-dire si l'autre peut continuer tout aussi bien que moi, c'est-à-dire continue tout à fait comme moi. » (Wittgenstein, 1983, § 45).

Cette unanimité n'est pas à considérer comme le produit d'un accord contractualisé (comme le présupposerait une vision naive du contrat) mais bien l'inverse : « on n'apprend pas à suivre une règle en apprenant d'abord l'usage du mot 'accord'. On apprend plutôt la signification d'accord' en apprenant à suivre une règle » (Schmitz, 1988, 246). Cette dimension collective est ici tout à fait essentielle car suivre une règle, reconnaître une preuve ou la nécessité d'une proposition, d'une démonstration... dépend d'une manière de voir qui n'a en soi rien d'inéluctable, car «Une démonstration n'en est une que pour celui qui la reconnaît comme telle. Celui qui ne la reconnaît pas comme telle, celui qui ne la suit pas comme démonstration, celui-ci nous quitte avant même que nous en discutions. » (Wittgenstein, 1983, I \& 61). Et c'est bien parce que les propositions mathématiques sont nécessaires (indépendantes du monde, non empiriques et normatives ${ }^{29}$ ) que les hommes s'imposent de les reconnaître identiquement (Schmitz, id., 243 et passim). Aussi, comme le fait remarquer Laugier (1998), il est fondamental de distinguer les deux sens 'd'accord' : « Dans le contrat (libéral) je suis la source, le point de départ de l'accord; mais je ne suis pas tout seul, donc nous nous mettons d'accord, moi et les autres. Dans la commu- nauté, je n'ai pas à me mettre d'accord : je le suis d'emblée, étant membre de la communauté. ».

Si l'on exigeait un fondement à ce que nous savons et à la manière dont nous devons nous conduire dans la communauté (le jeu et la manière de jouer), c'est probablement dans une anthropologie didactique qu'il faudrait aller le rechercher :

Les propositions mathématiques sont-elles des propositions anthropologiques qui disent comment nous, les hommes, inférons et calculons? - Un code juridique est-il un ouvrage d'anthropologie qui nous dit comment les gens de ce peuple traitent un voleur? etc. - Pourrait-on dire: "Le juge consulte un ouvrage d'anthropologie et condamne ensuite le voleur à une peine d'emprisonnement?" Mais le juge n'utiLisE pas le code comme un manuel d'anthropologie. (Wittgenstein, 1983, III, § 65).

D'où l'importance, comme le remarque Schmitz (ibid., 244), « d'un apprentissage spécifique qui se caractérise [...] par le fait que le 'professeur' traite d'une certaine manière les applications que l'élève fait d'une règle : il dit à l'élève ce qu'il doit obtenir et fait du résultat que l'élève obtient le critère de la bonne ou de la mauvaise application qu'il aura faite de la règle » et « toute tentative de justification ne pourra qu'aboutir à un 'c'est ainsi' qui n'est plus une 'raison'. S'il y a 'inexorabilité', sentiment de contrainte, etc. ce n'est pas parce que la règle contraint comme des rails contraignent la trajectoire de la locomotive, mais parce que nous agissons en étant inexorables. » (Schmitz, op. cit., 245 n. 28).

C'est entre la nécessité des propositions mathématiques et la contingence de sa reconnaissance que le contrat orchestre les illusions ostensives, dans une sorte de jeu du montré-masqué, mais qui, à terme, permettront à l'élève de s'inscrire dans une forme de vie. Comme nous l'avons écrit ailleurs (Sarrazy, 2002), les professeurs, comme les élèves, ont besoin à la fois de certitude et d'illusion; sans cela les premiers ne pourraient plus enseigner et les seconds ne pourraient pas apprendre. Lostension est probablement au nœud même de ce qui à la fois désenchante les professeurs, par faiblesse du 'montrer', et nourrit l'espoir et l'attente de l'élève par la force et le désir du 'caché' : une "pulsion scopique », conclurait, en référence à Freud $(1987,123)$, mon ami disparu à la mémoire duquel je voudrais ici rendre hommage, Gilles Dumas. 


\section{NOTES}

1. Gilles Dumas enseignait au Département des Sciences de l'Éducation de l'Université de Bordeaux 2. Il fut un membre assidu du DAEST et un des fidèles compagnons et témoin du travail de Guy et Nadine Brousseau au COREM. C'était très certainement l'un des meilleurs spécialistes du rapport entre la théorie des situations didactiques, la psychanalyse freudienne et la déconstruction derridienne.

2. Cet axe de recherche apparaît en tant que tel dans le programme de recherche du laboratoire de Didactique et d'Anthropologie des Enseignements des Sciences et Langagiers (le Daesl qui, depuis 2007 constitue une des équipe de l'EA 4140 : le LACES).

3. Ce programme ne peut ici être détaillé; le lecteur intéressé par ses fondements épistémologiques et théoriques peut se reporter à Sarrazy (2006); bien que partageant quelques références communes avec la théorie récente de l'action didactique conjointe (cf. Sensevy, Mercier, 2007), notamment dans les domaines de la pragmatique et de la philosophie du langage, il s'en distingue tout à la fois dans ses visées scientifiques mais aussi par une sensibilité épistémologique relative à la manière de considérer (et donc d'étudier) les positions et les fonctions des protagonistes de la relation didactique.

4. Nous éludons ici des étapes importantes (les rencontres de Lucienne Félix, de Colmez, de Lichnérowicz...) ; cf. les éléments de biographie de G. Brousseau exposés dans les premières pages de l'ouvrage Théorie des situations didactiques (1998).

5. On rappellera seulement qu'elle apparaît comme le produit d'un ensemble de processus résultant des interactions d'un sujet avec un milieu (cf. la distinction de Piaget entre « connaissance-état » et « connaissance-processus »).

6. Dont le titre initial était « Der Satz » (La proposition) ; c'est Moore qui, en référence au traité de Spinoza (Tractatus Theologico-Politicus), suggéra le titre à Wittgenstein.

7. Hormis un dictionnaire à l'usage des élèves de l'école primaire, c'est le seul écrit qui sera publié durant sa vie.

8. Durant sa captivité, Wittgenstein a rencontré un instituteur, Ludwig Hänsel, qui l'incita à se consacrer à l'enseignement.

9. Rappelons que Wittgenstein publia un dictionnaire à l'usage des élèves de l'école primaire mettant en avant-plan, selon sa propre expression, « le vocabulaire quotidien ordinaire. » (publié in Wittgenstein, 1986).

10. Brousseau n’a jamais évoqué Wittgenstein et pour cause, puisque Wittgenstein ne fut introduit en France que très tardivement et fut au moins jusqu'aux années quatre-vingt considéré comme un logicien. Lintérêt pour sa seconde philosophie n'est en fait que très récent.

11. Le concept de « disposition » n'est pas employé au sens bourdieusien, mais à celui qu'on lui accorde en philosophie de l'esprit (voir, par exemple, Engel, 1996).

12. Wittgenstein introduit pour la première fois le concept de forme de vie dans l'ouvrage majeur de sa seconde philosophie : les Investigations Philosophiques. Elles marquent une rupture avec la thèse tractuséenne de l'atomisme logique : comprendre une proposition ne consiste pas, comme il l'affirmait, à en comprendre ses parties constitutives mais à comprendre la manière de l'utiliser dans un langage; « Le parler du langage fait partie d'une activité ou d'une forme de vie. » (1961, § 23). La forme de vie pourrait se définir comme un ensemble de conditions non linguistiques qui constitue toute forme de compréhension des activités humaines: « La manière d'agir commune à tous les hommes est le système de référence à partir duquel nous interrogeons une langue inconnue. » (1961, § 23).

13. Si les connaissances pouvaient se communiquer explicitement, alors la plupart des théories didactiques seraient sans objet.

14. Ce ne sont pas bien sûr ces expressions en tant que telles qui posent problème, mais leur usage et ce à quoi elles sont associées (on sait tous que le soleil ne se lève pas...); en fait, il conviendrait de dire que "Tout se passe comme si... le professeur manipule le contrat » mais la rhétorique du raccourci laisse souvent le pas à l'erreur épistémocentrique dont parle Bourdieu, consistant à placer « au principe de la pratique [des agents], c'est-à-dire dans leur 'conscience', ses propres représentations spontanées ou élaborées ou, pire, les 
modèles qu'il a dû construire (parfois contre sa propre expérience naïve) pour rendre raison de leur pratique. » (Bourdieu, 1997, 65).

15. En effet, par la volonté didactique dont elle est porteuse, par sa force illocutoire (cf. par exemple les nombreux ou insistant « Regarde! » des professeurs dont « l'attention » constitue une des valeurs scolaires primordiales - et qui, probablement, trouve son énergie dans sa propre impuissance didactique, l'ostension pourrait, étonnamment, susciter le désir de savoir, un désir qui s'originerait dans ce manque que l'œil ne pourrait satisfaire. Le professeur montre, l'élève regarde mais ne voit pas. Pour Freud, la pulsion de savoir ou pulsion épistémophilique « travaille avec l'énergie du plaisir scopique. » $(1987,123)$. Cette thèse sera reprise par Lacan (Séminaire XI), et par Aulagnier pour qui « si tout désir de savoir est désir d'un savoir sur le désir, toute demande de savoir est demande de maîtrise sur ce champ du réel où l'autre paraît se mouvoir à sa guise, sûr d'y rencontrer les objets de son désir, alors que le sujet commence par le rencontrer comme champ du déplaisir, comme ce qui se dérobe et résiste. » $(1986,154)$. Ce savoir sur le désir apporte une maîtrise, un contrôle de l'incertitude attachée à une situation que Brousseau a exemplifiée, en référence à Freud, par le jeu du for-da (Brousseau, 1998; Brousseau, Otte, 1991).

16. On comprendra ainsi que c'est davantage l'ostension comme forme d'explication démonstrative dans la relation didactique qui nous intéresse ici dans ses rapports avec la thèse de l'indicibilité des relations sémantiques et non une réflexion sur la notion d'ostensif telle qu'elle est utilisée par exemple dans le cadre de la théorie anthropologique du didactique (TAD) ; la nuance est importante puisque la notion d'ostensif appelle nécessairement celle de l'ostension et de ses conditions de possibilité (il n'y pas d'ostensif en soi!). La question est donc importante mais ne peut ici être débattue sans risquer de diluer l'objet de ce texte : le lecteur curieux de ce débat peut consulter Sarrazy (2005). Je propose une analyse critique de la théorie anthropologique du didactique sur quatre axes : 1) la question des rapports technique-tâche 2) le statut praxéologique de la technique; 3) la conception et le rôle de la culture; 4) les conséquences anthropologiques et didactiques du dualisme de la TAD (opposition logos, techné; savoir, savoir-faire). Lidée est avancée qu'à ce dualisme est associé le risque d'un mentalisme conséquent à la désanthropologisation du didactique par l'oblitération des conditions de la genèse des connaissances et des techniques.

17. Conception courante chez les mathématiciens; Cantor, Frege ou Russell en furent certainement les représentants les plus illustres.

18. « Il n'y a pas de mathématique sans mathématicien », dit Apéry $(1982,62)$ et « les objets ne sont pas des entités absolues et intrinsèques, mais ils sont au contraire relatifs à l'activité des sujets " (Conne, 1999, 43).

19. Les règles du rugby fixent-elles la vitesse maximale du déplacement des joueurs? Peuvent-elles limiter l'intensité d'un plaquage? C'est de ces incertitudes que vient la nécessité d'un arbitre dont la fonction est précisément de décider arbitrairement de la conformité de l'action des joueurs à la règle lorsque celle-ci ne dit plus rien (cf. Jeudy, 1993).

20. Une règle, pour Wittgenstein, « se présente tel un poteau indicateur. [...] par quoi saurais-je interpréter son indication : dans la direction de la main, ou (p. ex.) dans une direction opposée [...] donc je puis dire que le poteau indicateur laisse subsister un doute. Ou plutôt parfois il en laisse subsister, parfois non » (1961, § 85).

21. Ces oppositions pourraient être travaillées pour distinguer les régimes épistémologiques de la Théorie anthropologique du didactique (TAD) et de la Théorie des situations didactiques TSD - on trouvera quelques éléments dans Sarrazy (2005).

22. « Nous avons été conduits à opposer 'ostension assumée' et 'ostension déguisée' pour rendre compte de procédés d'enseignement des mathématiques qui se sont développés depuis une dizaine d'années dans la scolarité obligatoire. Nous proposons donc de dire qu'un enseignement relève d'une présentation ostensive « assumée » : s'il n'existe pas au sein de la situation d'enseignement de situation a-didactique d'apprentissage; si le savoir, dont l'enseignement est visé, est présenté par le professeur dès l'entrée dans la situation didactique, puis « appliqué » dans des exercices proposés aux élèves. Nous avons proposé d'utiliser le terme 'd'ostension déguisée' quand l'enseignant, désireux de s'appuyer sur les connaissances antérieures des élèves, propose les exercices avant la présentation du savoir, et qu'il traite les réponses d'une certaine façon : il effectue un tri, parmi elles, pour valoriser celles qui conduisent assez rapidement au savoir visé sans prendre réellement en compte les autres. » (Salin, 1999). 
23. «L'explication ne finit jamais » écrit Wittgenstein (1961, 157). La reconnaissance de cet impossibilité de réduire l'altérité au même est une des conditions d'émergence du sujet; le didacticien s'accorde en cela avec le psychanalyste pour donner raison au pédagogue : le silence de l'un est nécessaire à la parole de l'autre. On peut aussi se reporter à Marchive (2007) qui montre remarquablement, a contrario de certaines positions dans les sciences de l'éducation, la fécondité et l'intérêt d'un rapprochement entre didactique et pédagogie.

24. « Il faut se garder de penser selon les catégories de la psychologie. Se garder, par exemple, de démembrer simplement l'expérience vécue en un voir et un penser, et autres choses semblables. » (Wittgenstein, $1985, \S 542)$

25. Sur cette question, on peut consulter le remarquable article de Fontaine (2001).

26. Cf. sur cet aspect Clanché, id., 1994.

27. En effet, pour Wittgenstein, comme pour Brousseau, la signification n'est jamais conçue comme un effet d'un contrat (au sens d'une convention) : « Nous sommes, dit Wittgenstein, à l'égard [de l'expression « $2+2=4 »]$ dans une situation qui est comparable à la situation que présuppose le Contrat Social. Nous savons qu'il n'y a pas eu de contrat effectif, mais tout se passe comme si un contrat de ce genre avait été passé. De même pour $2+2=4$ tout se passe comme si une convention avait été passée. » (1992, 190). C'est dans l'usage de la proposition, dans certaines circonstances qu'il faudra chercher la signification. Mais ceci ne signifie pas que le sens littéral, comme les savoirs du reste, 'n'existent' pas, mais c'est précisément du fait de leur existence qu'il est alors possible de les utiliser : « On pourrait parler d'un sens 'primaire' et d'un sens 'secondaire' du mot [songeons ici à 'savoirs' et 'connaissances']. Ce n'est que si le mot a pour vous un sens primaire que vous l'utilisez dans un sens secondaire. Ce n'est que si vous avez appris à calculer [...] que vous pouvez comprendre, au moyen de ce concept, ce qu'est le calcul mental. » (Wittgenstein, 1961, 349).

28. «Je montre à mes élèves des morceaux d'un immense paysage dans lequel ils ne seraient pas capables de se reconnaître. » (Wittgenstein, 1990, 73).

29. On peut consulter un remarquable travail empirique sur les formes d'avancée du temps didactique qui montre bien les effets de cette tension didactique entre une volonté didactique inspirée du constructivisme et les nécessités didactiques des milieux (Chopin, 2007).

30. « Le mathématicien invente toujours de nouvelles formes de représentation [...] [comme] un paysagiste qui crée des chemins pour un jardin : il se peut très bien qu'il les trace simplement comme des bandes ornementales sur la planche à dessin, sans penser un instant qu'un jour on les empruntera. [...] Le mathématicien ne découvre pas, il invente »(Wittgenstein, 1983, I, § 167-8).

\section{BIBLIOGRAPHIE}

Aebli H. (1966). Didactique psychologique : application à la didactique de la psychologie de Jean Piaget, 4 Éd., Neuchâtel : Delachaux et Niestlé, 163 p.

Apery R. (1982). Mathématique constructive, in J. Dieudonné, M. Loi, R. Thom et al., Penser les mathématiques. Paris : Seuil, 1982, 58-72.

Aulagnier P. (1986). Le « désir de savoir' dans ses rapports à la transgression. In P. Aulagnier. Un interprète en quête de sens. Paris, Ramsey. 145-160.

Bourdieu P. (1997). Méditations pascaliennes. Paris : Seuil. 316 p. coll. Liber.

Bouveresse J. (1987). La force de la règle, [Chap. 6, « Les propositions mathématiques ont-elles un contenu? »], Minuit, 1987, 69-85.

Brousseau G. (1972). Processus de mathématisation, La mathématique à l'école élémentaire, Paris : APMEP, 428-457.

Brousseau G. (1998). Théorie des situations didactiques, Grenoble : La pensée sauvage, 395 p., coll. Recherches en didactique des mathématiques.

Brousseau, M. Otte, (1991). The fragility of knowledge» In Mathematical knowledge : its grows through teaching, Kluwer academic press. 
Chauviré C. (1989). Ludwig Wittgenstein, Paris : Seuil, 280 p., coll. Les contemporains.

Chevallard Y., Johsua M.-A. (1991). La transposition didactique : du savoir savant au savoir enseigné, Grenoble : La pensée sauvage, $240 \mathrm{p}$.

Chopin M.-P. (2007). Le temps didactique dans l'enseignement des mathématiques. Approche des phénomènes de régulation des hétérogénéités didactiques. Thèse de doctorat de l'université Victor Segalen Bordeaux 2. (s/d du Pr. B. Sarrazy). 337 p.

Clanché P. (1994). L'enfant et le contrat didactique dans les derniers textes de Wittgenstein. In H. Hannoun, A.-M. Drouin-Hans, (Dir.). Pour une philosophie de l'éducation, [Actes du colloque " Philosophie de l'éducation et formation des maîtres Dijon, oct. 1993], Éd. CNDP, CRDP de Bourgogne, 1994, 223-232.

Conne F. (1999). Faire des maths, faire faire des maths, regarder ce que ça donne. In G. Lemoyne, F. Conne (dir.), Le cognitif en didactique des mathématiques. Presses de l’Université de Montréal. 31-69.

Conne F., Brun J. (1990). Début d'un enseignement, début d'un apprentissage: où placer les routines? [doc. Ronéo] Exposé de la journée du COED (Suisse).

Engel P. (1996). Philosophie et psychologie, Paris : Gallimard, 473 p.

Fabre M. (1999). Situations-problèmes et savoir scolaire. Paris : PUF, 1999, 239 p.

Fontaine O. (2001). Le « voir comme », entre « voir et penser» Remarques sur « l'espace grammatical » de la saisie d'aspects In C. Chauviré, S. Laugier, J.-J. Rosat (eds). Wittgenstein : Les mots de l'esprit. Philosophie de la psychologie. Paris : Vrin.159-182.

Fregona D. (1995). Les figures planes comme « milieu » dans l'enseignement de la géométrie : interactions, contrats et transpositions didactiques. Thèse de doctorat. Université de Bordeaux 1. 280 p.

Freud S. (1987). Trois essais sur la théorie sexuelle, Paris : Gallimard, 1987, 211 p., coll. Folio essais.

Jeudy H.-P. (1993). Éloge de l'arbitraire. Paris : PUF. 185 p.

Laugier S. (1998). Communauté, tradition, réaction, Critique, 610, 13-38.

Marchive A. (2007). La pédagogie à l'épreuve de la didactique : approche historique, recherches empiriques et perspectives théoriques. Presses Universitaires de Rennes (Soumis).

Monk R. (1993). Wittgenstein. Le devoir de génie, [traduit de l'anglais par A. Gerschenfeld], Paris : Odile Jacob, $628 \mathrm{p}$.

Piaget J. (1975). L'équilibration des structures cognitives : problème central du développement, Paris : PUF, $188 \mathrm{p}$.

Platon (1989). La République Livre VII In Euvres complètes, [traduction de L. Robin et M.J. Moreau], Paris : Gallimard, 1989, 1450 p., vol. 1, Encyclopédie de la pléiade, 1950.

Ratsimba-Rajhon H. (1992). Contribution à l'étude de la hiérarchie implicative : application à l'analyse de la gestion didactique des phénomènes d'ostension et des contradictions, Thèse de doctorat de l'Université de Rennes I, 210 p.

Rhees R., (1992). Commentaires sur la conférence sur l'éthique. In L. Wittgenstein, Leçons et conversations, Paris : Gallimard, 186 p., coll. folio essais.

Roiné C., (2007). La psychologisation de l'échec scolaire: une affaire d'état. Actes du Congrès International d'Actualité de la Recherche en Education et en Formation. 28 août au 31 août 2007. Strasbourg. http://www. congresintaref.org/prog.php

Rouchier A. (1996). Connaissances et savoirs dans le système didactique, Recherches en Didactique des Mathématiques, 16/2, 177-196.

Salin M.H. (1999). Pratiques ostensives des enseignants et contraintes de la relation didactique, In G. Lemoyne, F. Conne (dir.), Le cognitif en didactique des mathématiques. Presses de l'Université de Montréal. 
Salin M.-H. (2001). Les pratiques ostensives dans l'enseignement des mathématiques comme objet d'analyse du travail du professeur, In O. Venturini, C. Amade-Escot \& A. Terrisse, Étude des pratiques effectives : l'approche des didactiques, Grenoble : La Pensée Sauvage.

Sarrazy B. (1995). Le contrat didactique, [note de synthèse], Revue Française de Pédagogie, 112, 85-118.

Sarrazy B. (1997). Sens et situations : une mise en question de l'enseignement des stratégies méta-cognitives en mathématiques, Recherches en didactique des mathématiques, 17/2, Grenoble : La Pensée Sauvage, 135-166.

Sarrazy B. (2001). Les interactions maître-élèves dans l'enseignement des mathématiques : Contribution à une approche anthropo-didactique des phénomènes d'enseignement, Revue Française de Pédagogie. 136. 117-132.

Sarrazy B. (2002a). « Représentation»vs «modèle implicite» dans l'analyse des phénomènes d'enseignement : Analyse didactique et épistémologique des enjeux praxéologiques de cette distinction ». L'année de la recherche en sciences de l'éducation. Paris: PUF.

Sarrazy B. (2002b). Tribulations d'une utopie pédagogique en didactique ou les mésaventures de la transparence. Actes du Colloque International Utopies et pédagogies 27 au 27 mai Waldersbach.

Sarrazy B. (2005). Questions à la théorie anthropologique du didactique du point de vue de la théorie des situations et de l'anthropologie wittgensteinienne. Actes du Premier Congrès International sur la Théorie Anthropologique du Didactique. Société, École et Mathématiques: Apports de la TAD. Baeza (Jaén Espagne) du 27 au 30 octobre 2005 (A paraître).

Sarrazy B. (2006). Fondements épistémologiques et ancrages théoriques d'une approche anthropo-didactique des phénomènes d'enseignement des mathématiques. In G. Gueudet, Y. Matheron, Actes du Séminaire National de Didactique des Mathématiques. ARDM (Association pour la Recherche en Didactique des Mathématiques) et IREM Paris 7 : Jussieu. 21 octobre 2006. 79-99.

Sensevy G., Mercier A. (dir.) (2007). Agir ensemble. L'action didactique conjointe du professeur et des élèves. Rennes : Presses Universitaires de Rennes. 225 p.

Schmitz F. (1988). Wittgenstein et la philosophie des mathématiques. Paris: PUF. 281 p. coll. Philosophie d'aujourd'hui.

Solère-Queval S. (2002). La pédagogie invisible : Utopie ou réalité?. Actes du Colloque International « Utopies et pédagogies " 27 au 27 mai Waldersbach.

Wittgenstein L. (1961). Tractatus logico-philosophicus (Suivi des Investigations philosophiques), [traduit de l'allemand par P. Klossowski], Paris : Gallimard, 364 p., coll. TEL.

Wittgenstein L. (1965). Le Cahier bleu et le Cahier brun : Études préliminaires aux « investigations philosophiques » suivi de Ludwig Wittgenstein par N. Malcolm, [traduit de l'anglais par G. Durand], Paris : Gallimard, 1951, 423 p., coll. TEL.

Wittgenstein L. (1971). Carnets 1914-1916. [Traduction, introduction et note de G.G. Granger]. Paris : Gallimard. 249 p.

Wittgenstein L. (1975). Remarques philosophiques, [traduit de l'allemand par R. Rhees], Paris : Gallimard, 330 p., coll. TEL.

Wittgenstein L. (1976). De la certitude, [traduit de l'anglais par G. Durand], Paris : Gallimard, 152 p., coll. TEL

Wittgenstein L. (1983). Remarques sur les fondements des mathématiques, [traduit de l'allemand par M.A. Lescourret], Paris : Gallimard, 351 p., coll. Bibliothèque de Philosophie.

Wittgenstein L. (1985). Études préparatoires à la seconde parties des recherches philosophiques, [traduit de l'allemand par G. Granel], Mauvezin : Trans-Europ-Repress, 328 p., coll. T.E.R. bilingue.

Wittgenstein L. (1986). Vocabulaire à l'usage des écoles primaires. SUD, « Ludwig Wittgenstein », SUD, 1986, $16^{\mathrm{e}}$ année, numéro hors série. 
Wittgenstein L. (1989). Remarques sur la philosophie de la psychologie (I). [Traduit de l'allemand par G. Granel]. Mauvezin : Trans-Europ-Repress, coll. T.E.R. bilingue.

Wittgenstein L. (1992). Les Cours de Cambridge 1932-1935, [établis par Alice Ambrose à partir des notes d'A. Ambrose et de M. Macdonald, traduit de l'anglais E. Rigal], Mauvezin : Trans-Europ-Repress, 267 p., coll. T.E.R. bilingue

Wittgenstein L. (1993). Tractatus logico-philosophicus. [traduit de l'allemand et préambule de G-G. Granger. Introduction par B. Russell]. Paris : Gallimard. 121 p. coll. TEL.

Wittgenstein L. (1994). Remarques sur la philosophie de la psychologie (II). [Traduit de l'allemand par G. Granel]. Mauvezin : Trans-Europ-Repress, 142 p., coll. T.E.R. bilingue. 